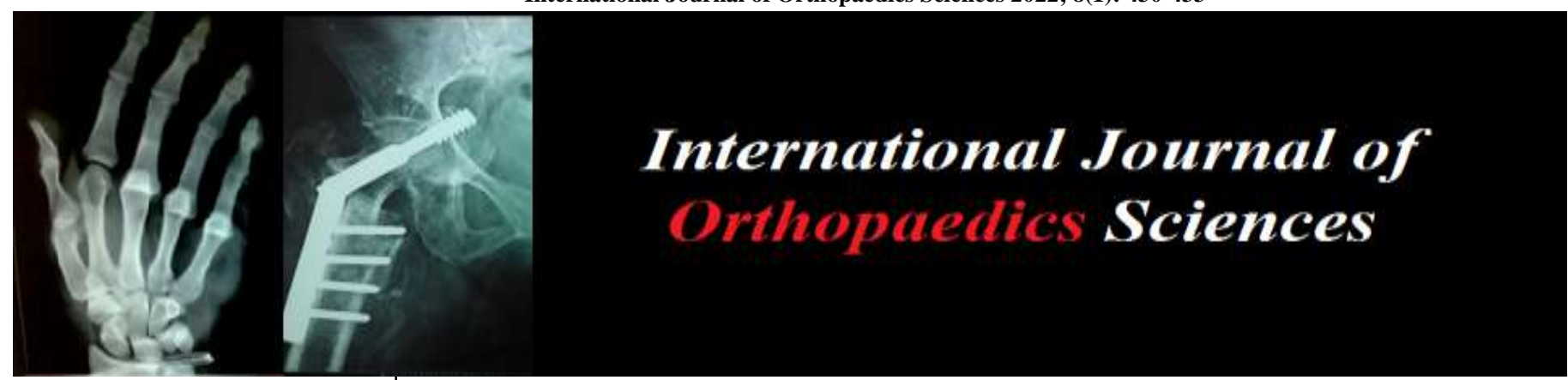

E-ISSN: 2395-1958

P-ISSN: 2706-6630

IJOS 2022; 8(1): 430-433

(C) 2022 IJOS

www.orthopaper.com

Received: 21-11-2021

Accepted: 26-12-2021

Dr. Vamshikrishna Chand

Nimmagadda

Assistant Professor, Department of Orthopaedics, VIMS \& RC,

Bangalore, Karnataka, India

Dr. Prajwal V

Junior Resident, Department of Orthopaedics, VIMS \& RC,

Bangalore, Karnataka, India

Dr. Manoj Kumar KCR

Junior Resident, Department of Orthopaedics, VIMS \& RC,

Bangalore, Karnataka, India
Corresponding Author: Dr. Manoj Kumar KCR Junior Resident, Department of Orthopaedics, VIMS \& RC,

Bangalore, Karnataka, India

\section{Effectiveness of platelet-rich plasma in the treatment of grade 1 osteoarthritis of knee}

\author{
Dr. Vamshikrishna Chand Nimmagadda, Dr. Prajwal V and Dr. Manoj \\ Kumar KCR
}

DOI: https://doi.org/10.22271/ortho.2022.v8.i1f.3052

\section{Abstract}

Background: The most common cause of arthritis of the knee is degenerative osteoarthritis (OA) which is one of the leading causes of disability all over the world. Population which is at higher risk of osteoarthritis are elderly females, patients with obesity, and African Americans. This is a concerning factor for the advancement and different modalities of treatment for early diagnosis and early intervention in degenerative and progressive joint disease of the knee. As a recent advance in treatment platelet-rich plasma (PRP) infiltration has come to the limelight for its advantages over others.

Purpose: To determine the effectiveness of platelet rich plasma in the treatment of grade 1 osteoarthritis of the knee.

Methods: Patients at Vydehi institute of medical science and research centre, Bangalore with radiologically confirmed grade 1 osteoarthritis of the knee were included after adequate counseling and consent. They were evaluated and followed up with Visual Analogue Score (VAS) and International Knee Documentation Committee (IKDC) scores at the end of 6 months.

Results: In a study of 100 patients with grade 1 osteoarthritis evaluated with Visual Analogue Score and International Knee Documentation Committee scoring at the end of 6 months with a mean score of 3.34 and 76.14 respectively. The study shows there is a positive correlation for the effectiveness of PRP in the treatment of grade $1 \mathrm{OA}$ of the knee.

Conclusion: PRP therapy is a simple, inexpensive, and minimally invasive intervention that is feasible to deliver in primary care to treat degenerative lesions of the articular cartilage of the knee. This therapy appears to have minimal associated adverse events and may have beneficial effects in terms of pain, health utility, patient satisfaction, and goal-orientated outcomes.

Keywords: Grade 1 osteoarthritis (OA), platelet rich plasma (PRP), intraarticular injection, hyaluronic acid (HA)

\section{Introduction}

The most common cause of arthritis of the knee is degenerative OA which is one of the leading causes of disability all over the world. Over 250 million people are affected by degenerative and progressive joint disease. Population which is at higher risk of OA (OA) are elderly females, patients with obesity, and African Americans ${ }^{[1]}$. Increased life expectancy and changing trends of lifestyle modification have the likelihood of incidence of OA in upcoming years are expected to be high in numbers. This is a concerning factor for the advancement and different modalities of treatment for early diagnosis and early intervention in degenerative and progressive joint disease of the knee.

$\mathrm{OA}$ of the knee is mainly classified into primary and secondary where primary are due to idiopathic or non-traumatic and secondary is due to, usually traumatic or mechanical misalignment. The severity of the disease has been graded using radiological findings by kellgren-Lawrence (KL) classification in $1957^{[2,3]}$.

As the disease is common, progressive, and increasing in incidence in recent times, it is important to diagnose and intervene in early stages. The treatment options for grade 1 OA have been growing from pharmacological methods which include analgesia, oral diacerein, glucosamine, chondroitin collagens etc. and non-pharmacological methods are using orthotics, physical therapy, or lifestyle modifications ${ }^{[3]}$. These modes of treatment have been vastly used, but have given short-term success, not treating the etiology or have shown minimal results. 
The new trend in treatment are the intra-articular injections which have been restricted to analgesics, steroids, hyaluronates, and some unproven alternative therapies which have given good results in a number of patients ${ }^{[4]}$. Hyaluronic acid and steroids have been the most common modes of treatment in intra-articular treatment options for the management of patients who fail to respond to nonpharmacological therapy and oral drugs. As a recent advance in treatment platelet-rich plasma infiltration has come to the limelight for its own advantages over others.

Platelet-rich plasma is prepared from autologous blood which is centrifuged in a way to obtain the concentration of platelets to a level above that normally found in serum, the PRP is a type of local cell therapy that is complex and comprises a large number of chemical mediators which interact with endogenous cells inside the joint ${ }^{[4]}$ and initiates immune responses for healing of the damaged cartilage.

\section{Materials and Methods}

This is a prospective study of 100 patients who were diagnosed with grade $1 \mathrm{OA}$ according to kellgren-Lawrence classification. All the patients involved in the study were picked among the outpatient department in Vydehi institute of medical sciences and research centre, Bangalore. The study was conducted between June 2019-December 2020 with a follow-up of 6 months. The outcome data were analyzed on the follow-up and was documented. The mean age of the patients, gender, BMI, and side involved are enumerated in Table-1.

The patients who were diagnosed with grade 1 degenerative and progressive joint disease of the knee were counseled regarding the study and consent was taken. A detailed history was taken with reference to knee joint $\mathrm{OA}$ and an examination of the knee was done. Plain Radiographs of the knee were taken as the criteria for diagnosis and grading of the disease was made.

\section{Inclusion criteria}

1. History of pain for a minimum of 4 months and not responding to NSAIDs or physical therapy.

2. Radiographic diagnosis of grade $1 \mathrm{OA}$.

\section{Exclusion criteria}

1. Higher grades of OA.

2. Non-degenerative arthritis of the knee.

3. Major axial deviation.

4. Haematological diseases.

5. Infections.

6. Immunosuppression.

Injection of intra articular PRP in knee joint technique ${ }^{[4]}$ The injection was given in the supine position and with all aseptic precautions-The affected side was exposed up to the thigh and cleaned with betadine scrub $(7.5 \%)$ and spirit. Then painted with $5 \%$ betadine solution and draped with linen towels. The knee joint was palpated and a good understanding of anatomical configuration was made.

$2 \%$ Xylocaine injection was given in the skin and soft tissues of the lateral aspect of the knee joint. The leg was held firm in neutral rotation and intra-articular injection of autologous PRP (platelet-rich plasma) was given by the lateral approach with knee incomplete extension using a 16gauge needle. After injection, patients were instructed not to use the injected leg for $24 \mathrm{~h}$, use ice packs over the injected joint, and not to use NSAIDs during this period.

\section{Preparation of autologous platelet-rich plasma (PRP) ${ }^{[4]}$}

The procedure consisted of $30 \mathrm{ml}$ of venous blood samples taken from every patient and collected in sterile sodium citrated tubes. Platelet concentrates rich in growth factors were obtained by the following technique: The tubes with citrated blood were centrifuged at $1800 \mathrm{rpm}$ for $15 \mathrm{~min}$ to separate erythrocytes, and at $3500 \mathrm{rpm}$ for $10 \mathrm{~min}$ to concentrate platelets. By this method, $5 \mathrm{ml}$ of PRP were obtained and injected immediately without storage. It has been stated that using freshly-harvested PRP might preserve all the platelet functions better.

The severity of pain was assessed by using the Visual Analogue Score (VAS) score and the international knee documentation committee (IKDC) score. The functional outcome was tabulated for each individual and the patterns of change were observed.

\section{Statistics}

Each patient has been evaluated thoroughly VAS and IKDC scores were documented at the time prior to the procedure and similarly evaluated at the end of 6 months. The values were combined to form a mean score at each time interval and The VAS score and IKDC were evaluated using paired T-test to know the statistical significance among them.

\section{Results}

Table 1: Demographic data.

\begin{tabular}{|c|c|c|}
\hline \multicolumn{2}{|c|}{ Total number of patients } & 100 \\
\hline \multirow{2}{*}{ Gender } & Male & 64 \\
\cline { 2 - 3 } & Female & 36 \\
\hline \multirow{2}{*}{ Age } & Range & $41-70$ \\
\cline { 2 - 3 } & Average & 31.6 \\
\hline \multirow{3}{*}{ BMI } & $18-24.9$ & $24 \%$ \\
\cline { 2 - 3 } & $25-29.9$ & $66 \%$ \\
\cline { 2 - 3 } & Above 30 & $10 \%$ \\
\hline \multirow{2}{*}{ Side } & Right & 64 \\
\cline { 2 - 3 } & Left & 36 \\
\hline
\end{tabular}

Table 1 depicts demographic data. A total of 100 grade 1 OA patients were taken up for study out of which males were 64 in number and females were 36 in number. Age distribution ranges from 41 to 70years with a mean age of 31.6years. patients with BMI of normal weight were $24 \%$ in number, overweight were $66 \%$ and obese were $10 \%$ of them. The study population showed right-sided dominance of 64 patients and 36 patients with left-sided involvement.

Table 2: Results.

\begin{tabular}{|c|c|c|c|}
\hline Scores & Baseline & 6 Month follow up & P-value \\
\hline VAS & $5.97+/-0.79$ & 3.34 & $<0.001$ \\
\hline IKDC & 38.56 & 76.14 & $<0.001$ \\
\hline
\end{tabular}

Table 2 depicts the functional outcome of 100 patients at a minimum follow-up of 6 months. Baseline scores of VAS and IKDC are 5.97+/- 0.79 and 38.56 and at the end of 6 months, the score improved to 3.34 and 76.14 respectively (Figure-1) which is statistically significant. 


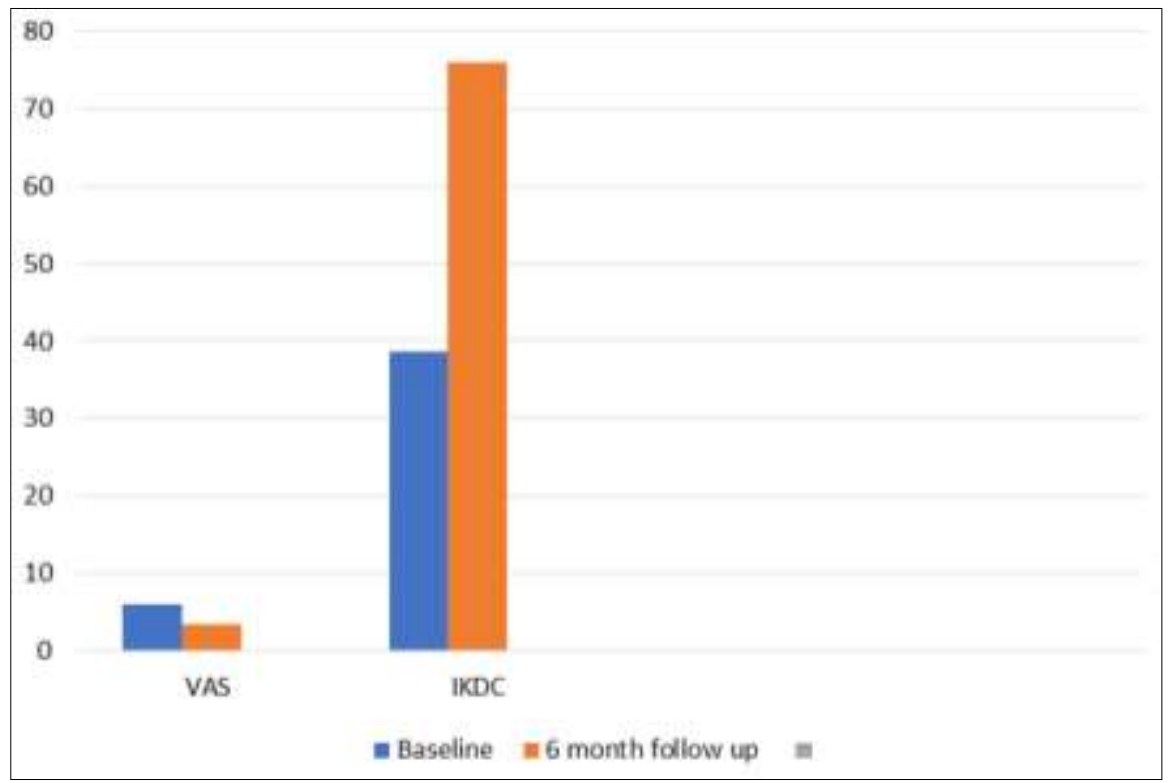

Fig 1: Mean scores of VAS score and IKDC at baseline and at 6 months follow up.

\section{Discussion}

In this study, it is demonstrated that PRP therapy in primary care of grade $1 \mathrm{OA}$ is feasible and safe. Follow-up and outcome measures were completed by all the patients. In our study, it has been shown that the primary measure of outcome i.e., pain, is significantly improved at the end of 6 months.

The treatment modalities for grade $1 \mathrm{OA}$ of knee referred to the conservative treatment, includes NSAIDs, analgesics, intra-articular hyaluronic acid injection, corticosteroid injection, weight loss, strengthening exercises.

Intra-articular PRP injection for symptomatic OA becomes the frequent mode of treatment. As it becomes a good promising agent of tissue repair and regeneration. Most studies involving PRP injection for OA shows improvement in pain and functional outcomes but lacks documentation. PRP-derived growth factors have been shown to promote cell recruitment, proliferation, and angiogenesis which results in the reduction of the critical regulators of the inflammatory process and results in decreased expression of inflammatory enzymes (Van Buul et al. 2011) [5]. Chondrogenesis and mesenchymal stem cell proliferation have been seen in PRP induced regenerative process of damaged tissue (Kabiri et al. 2014) ${ }^{[6]}$. In clinical practice, an appropriate scaffold is used along with autologous plasma and platelet-derived proteins to the desired location to assist in the repair of the injured tissue (Marx 2001) ${ }^{[7]}$. The rationale for use of scaffolds in PRP is to take advantage to promote cartilage regeneration by taking a huge amount of growth factors contained in platelets; however, the use of the PRP-augmented scaffolds is still in a preliminary state, which has a low scientific level of power (Kon et al. 2013) [8]. Growth factors promote matrix synthesis, migration and facilitate protein transcription and cell growth in chondrocytes application. The release of platelet-derived factors at the site of diseased cartilage directly, particularly in the case of knee OA. It may stimulate regenerative signaling cascade and enhance tissue healing with further anti-inflammatory response mediation (Mascarenhas et al. 2014) ${ }^{[9]}$. PRP showed to affect local and infiltrating cells, i.e. endothelial cells, those cells involved in innate immunity (such as macrophages) synovial cells, cartilage and bone cellular components (Mifune et al. 2013; Dhillon et al. 2017) [10, 11], in OA joints. PRP can affect inflammation, anabolic, angiogenic processes, and catabolic balance in cartilage formation. Additionally, alter the existing microenvironment during the progression of disease (Andia and Maffulli 2013) ${ }^{[12]}$.

Pilot and prospective studies investigating the clinical efficacy of intra-articular injections of PRP in patients with knee OA have demonstrated clinical improvement in selfreported pain and functional capacity with no major adverse effects ${ }^{[13]}$

A recent related systematic review included six randomized controlled trials comparing the effectiveness of PRP to other intra-articular injections, exercise, or analgesia for a minimum of 6 months. PRP injections were found to produce statistically significant improvements in overall WOMAC scores for patients with knee OA up to 12 months after intervention ${ }^{[14]}$.

Research into the efficacy of PRP has focused on comparing the effects of intra-articular PRP injections to other injection therapies. In many studies, PRP injections have improved functional outcomes when compared to HA and placebo controls and appear more efficacious in reducing symptoms and improving QoL (Raeissadat et al. 2015; Kanchanatawan et al. 2016) ${ }^{[15,16]}$.

Kon et al. (2011) ${ }^{[17]}$ examined three homogenous groups of patients treated with three injections of PRP, low molecular weight HA, and high molecular weight $\mathrm{HA}$ and concluded that autologous PRP injections have longer efficacy than HA injections and enhance articular function. The results showed improved outcomes for the PRP group at 6 months with younger and more active patients achieving better results with a low degree of cartilage degeneration (Meheux et al. 2016) ${ }^{[18]}$. Conversely, PRP causes a significantly greater acute inflammatory response and an increase in synoviocyte cell death (Braun et al. 2014) and induces more transient reactions than HA (Riboh et al. 2016) ${ }^{[19]}$.

The risk of adverse events in PRP-treated participants was not significantly increased in comparison with other knee OA treatment options ${ }^{[14]}$. These findings are consistent with much recently published research involving PRP as an intervention in knee OA ${ }^{[20]}$. The goal-oriented outcome approach used in this study has several advantages. It frames the discussion in terms of individually desired rather than universally applied health states; this approach simplifies decision making for patients with multiple conditions by focusing on outcomes 
that span conditions and aligning treatments toward common goals; goal-oriented care prompts patients to articulate which health states are important to them and their relative priority; and finally, if they know what health states are most desired, patients and clinicians can agree on steps that can be taken to achieve these goals and monitor progress in reaching them [21]. In essence, it allows the participant to co-design the outcomes based on their own individual preferences.

Despite the apparent positivity in the use of PRP for the treatment of knee OA, methodological concerns and considerable heterogeneity between studies are evident (Rodriguez-Merchan 2013b) ${ }^{\text {[22] }}$. Large RCTs are needed to further assess the efficacy and duration of PRP treatment for patients with knee OA (Rodriguez-Merchan 2013a; Lai et al. 2015) ${ }^{[23]}$. When planning or analyzing treatments, frequency and number of injections, as well as the activation methods (in the case of anti-coagulated PRP), storage aspects, time from plasma isolation, and accompanying therapy should be considered as at present they vary widely between groups. The greatest limiting factor for PRP use is the lack of standardization with further research required to investigate how leukocyte inclusion, activation, and platelet concentration affect therapeutic efficacy.

\section{Conclusion}

PRP therapy is a simple, inexpensive, and minimally invasive intervention that is feasible to deliver in primary care to treat degenerative lesions of the articular cartilage of the knee. This therapy appears to have minimal associated adverse events and may have beneficial effects in terms of pain, health utility, patient satisfaction, and goal-orientated outcomes.

\section{Conflict of interest}

The Authors declare no conflict of interest whatsoever arising out of the publication of this manuscript.

\section{References}

1. Mora JC, Przkora R, Cruz-Almeida Y. Knee OA: Pathophysiology and current treatment modalities. Journal of pain research. 2018;11:2189.

2. Hussain SM, Neilly DW, Baliga S, Patil S, Meek RM. Knee OA: A review of management options. Scottish medical journal. 2016Feb; 61(1):7-16.

3. Vaishya R, Pariyo GB, Agarwal AK, Vijay V. Nonoperative management of OA of the knee joint. Journal of clinical orthopaedics and trauma. 2016Jul 1; 7(3):170-6.

4. Jones IA, Togashi R, Wilson ML, Heckmann N, Vangsness CT. Intra-articular treatment options for knee OA. Nature Reviews Rheumatology. 2019Feb; 15(2):7790.

5. Van Buul GM, Koevoet WL, Kops N, Bos PK, Verhaar JA, Weinans $\mathrm{H}$ et al. Platelet-rich plasma releasate inhibits inflammatory processes in osteoarthritic chondrocytes. Am J Sports Med. 2011;39:2362-2370.

6. Kabiri A, Esfandiari E, Esmaeili A, Hashemibeni B, Pourazar A, Mardani M. Platelet-rich plasma application in chondrogenesis. Adv Biomed Res. 2014;3:138.

7. Marx RE. Platelet-rich plasma (PRP): What is PRP and what is not PRP? Implant Dent. 2001;10:225-228.

8. Kon E, Filardo G, Di Matteo B, Perdisa F, Marcacci M. PRP augmented scaffolds for cartilage regeneration: A systematic review. Oper Tech Sports Med. 2013;21:108115 .

9. Mascarenhas R, Saltzman B, Fortier L, Cole B. Role of plateletrich plasma in articular cartilage injury and disease. J Knee Surg. 2014;28:003-010.

10. Mifune Y, Matsumoto T, Takayama K, Ota S, Li H, Meszaros LB et al. The effect of platelet-rich plasma on the regenerative therapy of muscle derived stem cells for articular cartilage repair. Osteoarthr Cartil. 2013;21:175185.

11. Dhillon MS, Patel S, John R. PRP in OA knee - update, current confusions and future options. Sicot-J. 2017;3:27.

12. Andia I, Maffulli N. Platelet-rich plasma for managing pain and inflammation in OA. Nat Rev Rheumatol. 2013;9:721-730.

13. Paterson K, Nicholls $M$, Bennell K, Bates D. Intraarticular injection of photo-activated platelet-rich plasma in patients with knee OA: A double-blind, randomized controlled pilot study. BMC Musculoskelet Disord. 2016;17:67.

14. Mustafa A, Mallen CDM, Murphy AWM, Glynn LG. The effectiveness and safety of platelet-rich plasma intraarticular injections in the treatment of knee OA: a systematic review and meta-analysis of randomised controlled trials. Limerick: Health Research Institute Annual Scientific Meeting, 2017.

15. Raeissadat SA, Rayegani SM, Hassanabadi H, Fathi M, Ghorbani E, Babaee M et al. Knee OA injection choices: platelet- rich plasma (prp) versus hyaluronic acid (a oneyear randomized clinical trial). Clin Med Insights Arthritis Musculoskelet Disord 8: CMAMD, 2015, S17894.

16. Kanchanatawan W, Arirachakaran A, Chaijenkij K, Prasathaporn N, Boonard M, Piyapittayanun $\mathrm{P}$ et al. Shortterm outcomes of platelet-rich plasma injection for treatment of OA of the knee. Knee Surgery, Sport Traumatol Arthrosc. 2016;24:1665-1677.

17. Kon E, Mandelbaum B, Buda R, Filardo G, Delcogliano M, Timoncini A et al. Platelet-rich plasma intra-articular injection versus hyaluronic acid visco-supplementation as treatments for cartilage pathology: from early degeneration to OA. Arthroscopy: The Journal of Arthroscopic \& Related Surgery. 2011Nov; 27(11):1490501.

18. Meheux CJ, McCulloch PC, Lintner DM, Varner KE, Harris JD. Efficacy of intra-articular platelet-rich plasma injections in knee OA: a systematic review. Arthrosc J Arthrosc Relat Surg. 2016;32:495-505.

19. Riboh JC, Saltzman BM, Yanke AB, Fortier L, Cole BJ. Effect of leukocyte concentration on the efficacy of platelet-rich plasma in the treatment of knee OA. Am J Sports Med. 2016;44:792-800.

20. Shen L, Yuan T, Chen S, Xie X, Zhang C. The temporal effect of platelet-rich plasma on pain and physical function in the treatment of knee OA: systematic review and meta-analysis of randomized controlled trials. J Orthop Surg Res. 2017;12:16.

21. Reuben DB, Tinetti ME. Goal-oriented patient care-an alternative health outcomes paradigm. N Engl J Med. 2012;366(9):777-9.

22. Rodriguez-Merchan EC. Intraarticular injections of platelet-rich plasma (prp) in the management of knee OA. Arch Bone Jt Surg. 2013b;1:5-8.

23. Rodriguez-Merchan EC. Intra-articular injections of hyaluronic acid and other drugs in the knee joint. HSS J. 2013a;9:180-182. 